\title{
Sociologie de l'expérience lycéenne
}

\section{Mr François Dubet, Mr Olivier Cousin, Jean-Philippe Guillemet}

\section{Abstract}

The sociology of students experience. - Students experience is reviewed among eight senior high schools with differing social environment in Paris and its suburbs. About a hundred students and fourty teachers were interviewed. Though this population is not technically representative, we may say that it helps us to elaborate an image of students experience.

\section{Résumé}

Cet article présente de manière succincte une étude sur l'expérience lycéenne menée à la demande de la Direction des Etudes et de la Prospective du Ministère de l'Education nationale. II s'agit d'une intervention sociologique conduite auprès de huit groupes dans huit lycées hiérarchisés entre un « grand lycée » du Vle arrondissement parisien et des LEP de banlieue. Nous avons aussi réalisé une centaine d'entretiens auprès d'élèves et une quarantaine auprès d'enseignants. Cette population n'est nullement représentative, mais on peut considérer qu'elle permet de construire une image assez vraisemblable de l'expérience des élèves.

\section{Citer ce document / Cite this document :}

Dubet François, Cousin Olivier, Guillemet Jean-Philippe. Sociologie de l'expérience lycéenne. In: Revue française de pédagogie, volume 94, 1991. pp. 5-12;

doi : 10.3406/rfp.1991.1361

http://www.persee.fr/doc/rfp_0556-7807_1991_num_94_1_1361

Document généré le 07/06/2016 


\section{SOCIOLOGIE DE L'EXPÉRIENCE LYCÉENNE}

\section{par François DUBET, Olivier COUSIN, Jean-Philippe GUILLEMET}

Cet article présente de manière succincte une étude sur l'expérience lycéenne menée à la demande de la Direction des Etudes et de la Prospective du Ministère de l'Education nationale. II s'agit d'une intervention sociologique conduite auprès de huit groupes dans huit lycées hiérarchisés entre un " grand lycée * du V/Parrondissement parisien et des LEP de banlieve. Nous avons aussi réalisé une centaine d'entretiens auprès d'élèves et une quarantaine auprès d'enseignants. Cette population n'est nullement représentative, mais on peut considérer qu'elle permet de construire une image assez vraisemblable de l'expérience des élèves.

Longtemps en France, l'essentiel de la sociologie de l'éducation fut une sociologie sans acteurs. Les travaux fondateurs et majeurs portaient sur des flux, sur les effets agrégés des conduites, sur les fonctions de l'école, sur les " lois * du système sélectif, beaucoup plus que sur la manière dont les acteurs scolaires construisaient leur expérience, que sur leur * conscience *, aurait-on dit dans le vieux langage de la sociologie du travail. Un peu à la manière de ceux qui déduisaient la conscience ouvrière de la * condition * imposée par le capitalisme, l'expérience des élèves était inférée des logiques du système. Parce que le problème de l'inégalité des chances a été et reste encore le thème central de la sociologie de l'éducation française, l'élève a souvent été réduit à sa carrière et aux facteurs qui la fixaient.

II ne peut être question, bien sûr, de rejeter ces travaux et les questions qui les fondent, mais ils supposent une sorte de conformité et d'adéquation des acteurs au système, de part aveugle des conduites réduisant la subjectivité des élèves soit aux ruses de la raison, soit à la consolation ou à l'insignifiance. Le rôle proprement éducatif et socialisant de l'école peut étre alors ignoré, ramené à l'acquisition d'une position, soit encore à l'intériorisation, plus ou moins heureuse, d'une culture scolaire conçue comme un ensemble organisé et stable. Dans la mesure où le système scolaire se diversifie et se massifie, où il accroît son emprise sur l'adolescence et la jeunesse, dans la mesure aussi où les stratégies et les projets sont multiples, il importe d'étudier l'expérience scolaire des élèves eux-mêmes, de rechercher les orientations culturelles et les relations qui la fondent afin de savoir non pas quel acteur social fabrique l'école, mais quel acteur se fabrique dans l'école et comment.

\section{I. - QU'EST-CE QUE L'EXPÉRIENCE LYCÉENNE?}

1. La sociologie de l'éducation est tentée de concevoir les conduites des élèves à la lumière de modèles théoriques dérivant des représentations générales du système scolaire, et notamment de la production et de la reproduction des inégalités. L'expérience scolaire est alors réduite au principe central d'explication et d'analyse de ce modèle.

De manière dominante, les conduites des élèves, leurs choix, leurs subjectivités, leurs sociabilités, leurs modes d'adaptation, sont conçus comme l'expression d'une socialisation attachée à une position sociale. L'élève est porteur d'un "habitus" de classe plus ou moins proche des attentes culturelles et des modèles de rôle latents proposés par l'institution scolaire. L'espace de l'expérience scolaire est défini comme la rencontre d'une culture sociale, d'une culture de classe et d'une culture scolaire. Les attitudes des élèves dérivent de la distance de ces deux cultures et des systèmes de dispositions qu'elles engendrent et qu'elles requièrent. Les héritiers décrivent tout le jeu de connivences et de sousentendus qui organise l'expérience des élèves prédisposés à gérer la rencontre de deux cultures proches. A contrario, l'échec scolaire est expliqué par la distance de ces cultures et par l'intériorisation de la difficulté de réussir provoquée par cette distance, si forte parfois que les sociologues britanniques ont pu opposer la culture scolaire et la sous-culture déviante des élèves issus des classes populaires (1). Ici, le problème de la massification relative du public des lycées se ramène principalement à 
la confrontation de nouvelles attentes et dispositions avec le modèle culturel scolaire proche de celui des classes dominantes. Mais pouvons-nous réellement, dans ce cadre, considérer l'élève comme un acteur social ? II ne semble pas, dans la mesure où les conduites des sujets paraissent totalement déterminées par le jeu des positions sociales. Plus exactement, tout se résume dans ce jeu des positions où les conduites des acteurs semblent nécessaires, où l'expérience scolaire n'a pas de logique propre, où la subjectivité n'est qu'un effet des positions sociales.

Contre cette représentation de l'élève, le courant de l'individualisme méthodologique considère l'élève comme le sujet d'un marché où se croisent les biens scolaires et les ressources des acteurs percus alors comme des stratèges et des consommateurs. Au lieu d'être le support d'attitudes culturelles déjà là, l'élève est un stratège qui optimise ses investissements et ses coûts, choisit la meilleure solution à chacun des carrefours que lui impose le système scolaire (2). L'expérience de l'élève est celle d'un agent économique face à la rareté relative des biens offerts, des diplómes et des positions sociales, des jeux d'inflation et de concurrence qui fixent les hiérarchies et les filières. Si l'acteur social existe ici, il est réduit à une position de stratège, et la fonction proprement socialisatrice et éducative de l'école est ramenée à un principe d'intérêt ou, plus souvent, passée sous silence.

Il est bien évident que ces deux modèles correspondent à deux cadres théoriques antinomiques, à deux représentations de l'école. Mais elles sont aussi peut-être associées à deux "åges * de l'institution scolaire en France. La première considère l'école comme une institution organisée autour d'un modèle culturel central et dominant, proche de l'image « classique » du lycée, la seconde conçoit l'école comme une série de filières, de carrefours; plus sensible à l'état actuel du système scolaire, l'école y est moins perçue comme une institution de socialisation à la manière de Durkheim, que comme un marché de biens scolaires, l'étude des rapports de concurrence efface celle des biens objets de cette concurrence (3). De l'institution au marché, il n'y a pas seulement deux cadres théoriques, mais aussi deux définitions du rôle de l'école.

II faut dire un mot de ce que l'on a appelé " la nouvelle sociologie * anglaise de l'éducation, inspirée par l'interactionnisme symbolique et l'ethnométhodologie, et qui est plus sensible à l'idée d'expérience scolaire (4). Le point original de cette perspective, pour peu qu'on lui accorde une unité, est l'analyse des interactions dans la classe et notamment des relations entre les maîtres et les éleves. Les travaux montrent comment les enseignants attribuent des rôles, étiquettent les élèves et comment ceux-ci résistent et réagissent à ces diverses interactions.
Mais conçue comme une série de relations, l'expérience scolaire semble ne plus avoir d'unité propre et de principes centraux et, la plupart du temps, cette sociologie a considéré l'expérience des élèves comme un sous-système de la première perspective évoquée ici, celle de la rencontre culturelle.

2. L'idée d'expérience scolaire suppose que l'on ne considère pas seulement l'élève comme un individu face à une situation et des contraintes, mais comme un acteur construisant son expérience à travers des choix culturels et des orientations, élaborant des stratégies et les significations de ses stratégies dans un système de relations sociales. II importe de dégager les dimensions centrales de cette expérience, celles que l'acteur doit gérer et articuler.

La première de ces dimensions est celle du projet. Chaque élève entretient nécessairement avec l'école un rapport d'utilité lié à un projet. II faut que les études et le travail aient un sens par rapport à l'image qu'il se fait de son avenir; ce projet peut avoir une dimension professionnelle, il peut au contraire se limiter à une carrière scolaire, passer dans la classe supérieure. Ce projet peut être vécu par l'élève comme étant le sien propre, ou bien comme celui d'un quasi-destin familial dans lequel les études s'imposent comme allant de soi. De ce point de vue, l'absence de projet, par les problèmes qu'elle pose quant aux "motivations " des élèves, relève de la même logique de l'action, celle qui implique nécessairement que les études aient un sens et une utilité. Leur défaut est perçu comme une quasi-pathologie.

Mais un élève ne peut pas être seulement défini par un projet car il vit la rencontre de ce projet et d'une organisation. En effet, une école est aussi une organisation définissant des róles, des conduites, des statuts, des voies obligées. Généralement, la rencontre de ce projet et de l'organisation se noue autour de la sélection et de la mesure des performances scolaires. Chaque élève est membre d'une organisation sociale et d'une communauté juvénile dont les capacités d'intégration sont plus ou moins fortes, dont les définitions de rôles sont plus ou moins contraignantes et dont les espaces d'autonomie sont plus ou moins grands.

Enfin, un élève est aussi un acteur culturel. Les connaissances acquises se limitent au cadre scolaire ou le débordent, mais elles proposent une image de soi et du monde, elles ne se réduisent pas à de simples supports de classements sans contenu. En tout cas, les élèves peuvent construire plusieurs orientations à l'égard de ces connaissances et des enseignants ; ils peuvent choisir une logique instrumentale où l'évaluation des connaissances commande leur apprentissage, ils peuvent soumettre cet apprentissage à la seule relation pédagogique, " On travaille pour le professeur ", ils peuvent enfin déve- 
lopper un rapport d'intérêt intellectuel vis-à-vis de certaines disciplines. Quoiqu'il en soit, ce rapport à la connaissance participe de la fonction éducative de l'école et de la formation d'un certain type d'acteur social.

3. Si l'on se place du point de vue des acteurs, l'expérience scolaire n'a guère d'unité car chacune de ses dimensions est en tension avec les autres. Le projet personnel ou social avec sa charge d'utilité ne sont pas toujours en adéquation avec la logique de l'organisation et de la sélection. Bien souvent même, l'école apparaît comme un obstacle à ce projet et l'élève doit sans cesse réajuster ses ambitions et ses désirs aux opportunités offertes et aux ressources dont il dispose. Par ailleurs, il se crée une tension entre les nécessités de l'intégration et de la sélection d'une part, et les objectifs culturels de l'enseignement de l'autre, qui se joue dans les relations proprement scolaires, comme dans la vie juvénile abritée par le lycée.

Dès que l'on abandonne l'univers relativement intégré et harmonieux des * bons élèves * des * bons lycées *, où l'utilité des études, leur fonction culturelle et l'intégration scolaire de l'élève s'accordent de manière harmonieuse, où le * style * même devient central, dès que l'on délaisse en fait le petit monde des "héritiers", l'expérience scolaire apparaît comme beaucoup plus éclatée, plus diversifiée, plus chaotique, et c'est alors qu'il faut étudier les logiques des acteurs, la façon dont ils construisent cette expérience et se construisent eux-mêmes. L'élève est face à un projet, face à une organisation des études et face à une activité culturelle dont rien n'indique qu'elles forment système et qu'elles définissent un rôle d'élève auquel il suffirait d'être conforme. Dans le cas de ces "nouveaux lycéens", les conduites des élèves ne peuvent plus être directement inférées du système.

Plus on s'éloigne du * cœur * du système scolaire capable d'intégrer les fonctions de l'école, plus il devient nécessaire d'étudier l'expérience des élèves et les logiques de leur action car moins l'école y apparaît comme un système unifié. Tout s'y passe comme si les logiques sélectives, la capacité d'adaptation à l'environnement et le role éducatif de l'école se croisaient au lycée sans jamais réellement s'intégrer. L'acteur se trouve donc * obligé * de construire son expérience, ne pouvant plus se laisser porter par les * lois * du système ou ses seuls intérêts stratégiques.

\section{II. - LES PUBLICS DES LYCÉES}

De manière extrémement grossière, trois grands publics scolaires peuvent être distingués en fonction des établissements, des groupes sociaux dont sont issus les éléves, et des carrières scolaires qui leur sont offertes.
Evidemment, cette classification reste très éloignée de la multiplicité des publics rencontrés lors de notre étude et nous ne la retenons ici que pour des commodités d'exposition.

\section{Les * bons élèves * des * bons lycebes *}

Si l'on excepte les grands lycées parisiens dominés par les classes préparatoires, disposant de la possibilité quasi totale de choisir leur public et recrutant dans de véritables généalogies familiales, le style "héritier " semble résiduel même s'il reste au cour des représentations. L'aisance aristocratique, la distance critique à l'égard du " scolaire n, la certitude de la réussite, l'esprit de caste et la construction d'une distance intellectuelle ne semblent plus étre le centre des conduites de la plupart des lycéens.

La plupart des " bons élèves " des "bons lycées *, des " vrais lycées * traditionnels des centre-villes, entretiennent un rapport plus instrumental et plus tendu à leurs études. Le bac n'y apparaît ni comme un passeport vers la liberté des jeunes adultes, ni comme le premier écheIon d'une mobilité. Inscrits dans une sorte de destin scolaire et d'obligation familiale de réussite, les études se présentent comme un jeu où les risques de pertes sont plus élevés que les chances de gains; tout se passe comme s'il fallait obtenir un diplóme qui ne vaut plus grand chose. Les projets professionnels et les a vraies vocations * scientifiques ou littéraires apparaissent comme extrêmement rares ; l'âge des choix se présentera après le bac et le projet scolaire est étroitement lié à la carrière scolaire elle-mème, il faut passer dans la classe supérieure. Les élèves développent une logique du a diffèrement " où les * vraies " questions se poseront plus tard, où la * vraie "vie commencera après, lors des études supérieures.

Cette faiblesse des projets doit être mise en rapport avec les mécanismes de la sélection. Si les grands lycées parisiens restent encore partiellement organisés autour de l'opposition des cultures et des stéréotypes littéraires et scientifiques, grâce à la constitution volontaire de bonnes classes littéraires A1 capables de rivaliser avec les C, il n'en reste rien dans la majorité des établissements. La hiérarchie des filières répartit les qualités scolaires plus que les goûts des élèves. De la filière $C$ à la filière $A 2$ ou A3, selon les établissements, se répartissent les vertus scolaires des élèves, et les * vrais scientifiques " comme les « vrais littéraires " sont une minorité. II se crée alors une tension constante entre les goûts affirmés et les filières, attribuées plus que choisies, malgré les faibles dénégations des élèves.

Cette tension, largement décrite par les enseignants qui la condamnent et la perpétuent tout à la fois, asso- 
ciée à l'absence de projet professionnel précis, entraine un rapport très utilitariste aux études, complètement dominé par un principe d'efficacité, voire de cynisme. Malgré la présence d'enseignants jugés exceptionnels par leur talent et leur enthousiasme, les élèves choisissent avant tout l'efficacité du professeur, aux dépens de sa capacité de stimuler l'intérét intellectuel et de la chaleur des relations qu'il peut entretenir avec la classe. Les élèves se présentent alors comme des virtuoses de l'efficacité, de l'adaptation adéquate, de la justesse des investissements en fonction des coefficients, de la capacité de répondre aux attentes diffuses. Les lycéens se présentent comme des entrepreneurs rationnels, efficaces parce que distanciés et détachés, refusant de s'engager au-delà du nécessaire.

D'après les élèves, si les études peuvent avoir un rôle dans la formation de la personnalité, un rôle éducatif, c'est par la rencontre miraculeuse d'un enseignant hors du commun et, plus encore, par la vie juvénile offerte par le * bon lycée *. Chaque classe est organisée en * clans * fondés sur des goûts, des styles, des aspirations, et chacun d'eux apparaît comme le véritable espace de la vie personnelle et intellectuelle. C'est là que les élèves pratiquent ce que l'un d'eux nomme l'k art de la conversation ॠ, cette vie juvénile nichée dans les temps morts de la vie scolaire et pratiquant à des degrés divers une sorte d'éthique de la non-compromission avec la vie et la culture scolaire, comme si la personnalité devait étre préservée de l'école. Alors que dans les grands lycées la compétition et la performance sont au centre de la vie scolaire, la grande majorité des élèves "vise la moyenne * et une adaptation peu engagée dans l'école.

Ces stratégies d'adaptation, avec leur éclatement et leur dispersion, sont d'autant plus nettes que la plupart des lycées ont une très faible capacité d'intégration. Le lycée propose une succession de cours mais il n'exerce guère de contrôle social; au contraire, les élèves perçoivent l'établissement comme une organisation extrêmement libérale, parfois anarchique, autorisant une vie juvénile autonome, la multiplicité des looks, une vie amoureuse et amicale libre, méme si le prix de ce libéralisme est l'absence de participation et, nous le verrons, l'absence de droits, l'impossibilité de construire des conflits et de peser sur l'enseignement et l'organisation. Selon les élèves, on est au plus loin du * lycée caserne ", il s'agit plutôt d'une bureaucratie offrant des espaces de liberté personnelle; ainsi, il n'y a guère de problèmes de discipline et de chahut, on y parle surtout d'absentéisme et de manque d'intérêt. II est vrai que dans la plupart des établissements étudiés, les CPE et l'administration se montrent accommodants et modèrent un face à face trop brutal entre les enseignants et les élèves. De méme que les familles de classes moyennes supérieures proposent à leurs enfants d'échanger une liberté personnelle et juvé- nile contre une performance scolaire convenable, l'établissement échange une efficacité scolaire contre une vie juvénile peu contrainte.

\section{Les nouveaux byctens}

II s'agit des élèves des sections dévalorisées, notamment de sections $G$ et $F$, fréquentant les nouveaux lycées construits souvent dans les banlieues populaires, des élèves dont les parents n'ont souvent pas fait d'études secondaires longues, bref, des élèves qui préfigurent les gros bataillons des fameux \& $80 \%$ * de bacheliers.

Qu'ils aient des projets professionnels ou non, qu'ils envisagent des études supérieures ou pas, ces élèves sont pris dans une situation paradoxale. Ils s'apparentent aux boursiers dans la mesure où le fait même de poursuivre des études secondaires longues les place, à l'égard de leur famille, sur une voie de mobilité ascendante. Ils seront souvent les premiers bacheliers de leur famille et ce parcours est particulièrement net pour les enfants d'ouvriers et d'immigrés qui composent de larges tranches de cette population. Cependant, ces élèves en montée sociale sont en chute scolaire. Contraints de choisir les sections et les établissements " poubelles *, c'est ainsi qu'ils les nomment, comme un grand nombre de leurs enseignants, ils éprouvent un sentiment de dévalorisation et de honte lorsqu'ils se définissent à l'égard des normes de l'excellence scolaire. Ils ne peuvent même plus se consoler gråce à l'ancienne dignité des sections littéraires. Aboutissant dans ces sections par une scolarité cahotique, ils sont souvent âgés et se sentent piégés par un diplơme fermant plus de portes qu'il n'en ouvre.

Le modèle culturel proposé à ces élèves s'appuie sur les enseignements concrets, comptabilité, gestion, etc. Mais ces enseignements sont scolairement dévalorisés puisque dévolus aux élèves les plus faibles. Les enseignements généraux subsistent sans que les élèves en perçoivent l'utilité, ils les entendent comme des réponses à des questions qui n'ont jamais été posées et, souvent, comme les moyens de perpétuer l'échec. Ne pouvant s'appuyer sur un intérêt intellectuel valorisé, ces élèves sont tentés d'opposer la " vie réelle et concrète " à l'abstraction des études. Cette vie réelle l'est d'autant plus que les élèves sont âgés et vivent souvent comme de jeunes adultes, travaillent pour leur argent de poche ou pour leur entretien, alors que la dévalorisation du diplóme en fait un investissement peu rentable et cependant indispensable.

Ces attitudes, ces contradictions et ces paradoxes conduisent les élèves vers une grande dépendance à l'égard des enseignants. Eloignés de l'intérêt intellectuel, peu capables de développer des stratégies efficaces, ils se perçoivent comme très dépendants de l'enseignant et 
de sa personnalité. Alors que les "bons élèves des bons lycées * sont sensibles à l'efficacité des enseignants, les nouveaux lycéens sont d'abord sensibles à leur * personnalité *. Les cours apparaissent comme des successions de relations interpersonnelles où le mépris des enseignants le dispute à la gratification et aux encouragements. Ces nouveaux lycéens développent alors une sorte de pensée * magique ", * on peut si on veut ", et oscillent sans cesse de l'espoir au découragement. Souvent conformistes, ils ne parviennent pas à maitriser efficacement le jeu qui leur est proposé.

Cette dépendance malheureuse est le plus souvent vécue dans le mépris et l'humiliation, celle des établissements * mal fréquentés *, celle des professeurs parfois déçus d'enseigner à des élèves faibles, celle qui est attachée à un passé scolaire suffisamment difficile pour dévaloriser l'image de soi. Même des élèves des classes d'adaptation, issus des LP et qui ont le sentiment de se sauver, ne résistent guère à ce climat dépressif.

Comme ces " nouveaux * lycées sont des établissements libéraux et peu contraignants, la vie juvénile s'y développe aussi fortement que dans les "bons lycées *. Toutefois, elle ne constitue nullement une communauté délinquante; elle est tout simplement en dehors de la vie et de la culture scolaire. Au plus loin des exigences de travail et de continuité, de * gratuité * des exercices scolaires abstraits, elle se constitue autour de la " vie réelle », vie difficile souvent, ou vie de loisirs et de sorties dont les élèves expliquent qu'elle redonne une image positive de soi. Entre l'école et la "vie ", ces lycéens perçoivent parfois la méme distance que celle des ouvriers opposant l'usine à cette vie.

\section{Les Glives de LP}

Bien que le recrutement des élèves soit socialement plus homogène que celui des * nouveaux lycéens *, le monde des LP paraít clivé et l'expérience sociale des élèves peut être comprise comme l'expression de ce clivage (5).

La première ligne de fracture oppose l'enseignement général à l'enseignement professionnel. Non seulement les élèves ont affaire à deux populations d'enseignants aux styles culturels tranchés, mais ils ont le sentiment de se trouver dans deux écoles. L'enseignement général, malgré de considérables efforts des professeurs, perpétue une relation pédagogique traditionnelle où la contrainte physique de silence et de discipline reste forte et où l'attention est concentrée sur le professeur. Au contraire, l'atelier laisse une autonomie physique de déplacement et d'attention et centre l'attention sur une táche plus que sur un enseignant. Quelles que soient leurs performances, les élèves ne parviennent pas à surmonter totalement cette dualité.
Le second axe de clivage oppose les élèves de CAP et de BEP dévalorisés aux élèves préparant un bac professionnel ou un BEP qualifié. Les premiers élèves aboutissent au LP faute de mieux, sans projet professionnel, et souvent hostiles à l'école. Leur expérience est avant tout décrite en termes de sociabilité juvénile et souvent de communauté délinquante. Le LP ne parvient pas à socialiser les élèves à ses normes propres et les cours et les professeurs sont jugés en fonction de l'espace qu'ils donnent à cette sociabilité. Comme il n'est pas un univers disciplinaire et comme les enseignants essaient de s'adapter le moins mal possible à cette société qui leur échappe, le LP prolonge souvent la vie du quartier, de la bande et de la " galère *.

Contrairement aux anciens collèges d'enseignement technique qui proposaient une socialisation à une culture ouvrière du métier et à une discipline industrielle (6), le LP abrite une jeunesse qui n'a pas d'espoir de mobilité et pour laquelle l'école n'engendre souvent que de l'échec. L'enseignement est perçu comme une myriade de rencontres avec des personnalités diverses, plus ou moins ouvertes ou rigides, définies avant tout par leur capacité d'imposer une discipline. De leur cóté, les enseignants ne parlent guère de ces élèves en termes intellectuels ou strictement scolaires, ils les définissent d'abord en termes de psychologie et de comportement.

Issus de la longue sélection qui s'opère dans les classes de CAP et de BEP dévalorisés, les élèves des sections préparant au bac professionnel ou aux bons BEP entrent dans une expérience totalement opposée à celle des précédents. Ils parviennent mieux à harmoniser les deux cultures scolaires, ne serait-ce que parce qu'ils rencontrent des professeurs d'enseignement professionnel qualifiés, les PLP 2, légitimes dans les deux univers culturels et identifiés à un monde de techniciens. La fierté professionnelle est aussi renforcée par le fait que les sections préparant au bac professionnel sont l'image de marque valorisée de l'établissement. Au bout du compte, ces élèves ont l'impression de " s'en sortir * et sont placés au sommet du système d'enseignement professionnel, ils se définissent positivement par leurs techniques et leurs compétences. II faut opposer leur expérience à celle des élèves des sections $G$ qui sont, eux, " en bas " des références scolaires classiques. Par ailleurs, ces élèves relativement ágés se comportent comme des jeunes adultes entrant déjà, par le biais des stages, dans la vie active.

L'expérience scolaire des élèves de LP apparait donc tendue entre les deux póles constitués par une sociabilité juvénile mal enserrée dans le cadre scolaire et une formation professionnelle accordée à une sorte d'élite professionnelle. Ceci n'empeche pas l'ensemble de cet univers d'être sensible, plus que les autres, au marché de l'emploi, et tandis que les premiers élèves pensent en 
termes d'emploi, n'importe lequel, les seconds pensent, eux, en termes de métier. De manière un peu excessive sans doute, on pourrait dire que le LP oscille entre le traitement social du chómage des jeunes et la formation de professionnels qualifiés.

\section{III. - L’élève et L'ÉCOLE}

Au-delà de ces différences sensibles entre les divers publics scolaires, il est possible de construire un portrait général de l'expérience lycéenne. Cette expérience, essentiellement organisée autour d'un processus de sélection qui conduit les jeunes à adopter des stratégies très fortement empreintes d'instrumentalisme, se traduit par la dissociation de la vie culturelle et de l'école, par la faible capacité de socialisation de l'institution, par la séparation de la personne et du système.

1. La massification du système scolaire engendre une diversification des filières qui génère une hiérarchisation des différentes sections. Chaque élève sait bien aujourd'hui que tous les bacs ne se « valent » pas. En distribuant des positions scolaires différentes et donc inégales, l'école est perçue comme une immense * gare de triage $\nsim$, une gigantesque « machine à sélectionner *. Le parcours du lycéen est en effet jalonné de points de bifurcation, au cours desquels le système évalue les performances de chacun et les sanctionne par une orientation ou un examen. La sélection imprègne si fortement l'expérience de ces jeunes que ce thème domine constamment les premières réunions des groupes d'élèves et les entretiens. Les élèves commencent d'ailleurs toujours par affirmer l'absence de hiérarchie entre filières. Les différentes sections sont présentées comme * différentes mais égales », et chacun déclare avoir choisi son orientation. Ce discours, qui est le fruit d'un processus de réduction de la dissonance psycho-sociale, exprime également, sur le plan sociologique, le refus unanime de briser la fragile communauté des élèves. Mais il se brise très vite et les lycéens introduisent toujours, parfois de manière très douloureuse, les thèmes de la sélection et de la hiérarchie. En effet, à l'exception de la première $S$ et de la terminale $\mathrm{C}$, les élèves ne choisissent pas leur orientation, et ceux qui se trouvent dans la voie royale n'y sont pas toujours par goût. Ils essaient surtout d'effectuer le moins mauvais " choix possible *. Les lycéens s'accordent d'ailleurs très rapidement sur la hiérarchie des filières : $C, D$, A1, B, A2, A3 (chaque établissement peut aménager cette hiérarchie, notamment entre $A$ et $B$ ), les diverses sections $F$, puis les $G$, hiérarchisées, enfin les * bacs pro * et les BEP, également hiérarchisés. Ils n'hésitent que sur la place à accorder au bac $E$, dans la mesure où les études techniques ne jouissent pas toujours d'une bonne image. Les élèves connaissent en outre parfaitement les clés de la sélection et ils savent qu'elle commence dès le collège par le choix des langues en $6^{\circ}$ et en $4^{\circ}$, l'importance de l'allemand, du latin et du grec... Si la plupart d'entre eux ne réussissent pas dans ce système sélectif, ils n'en sont donc pas pour autant aveugles et tentent de s'y adapter. A la hiérarchie des sections se superpose de plus une image très précise de la hiérarchie des établissements. II y a de bons et de mauvais lycées classiques, de bons et de mauvais lycées techniques, de bons et de mauvais lycées professionnels. Tout comme on ne choisit pas son orientation, on ne choisit pas toujours son lycée. Plus on descend dans la hiérarchie des sections et moins l'on choisit: "On va là où l'on est accepté ». Les parents des meilleurs élèves, souvent avec la complicité passive de l'administration, n'hésitent d'ailleurs pas à contourner l'obstacle de la carte scolaire pour éviter le * lycée dépotoir * du secteur, où les sections $C$ cohabitent avec les sections $G$. Un bac identique ne possède pas la mème valeur selon l'établissement dans lequel il est préparé. La reconnaissance de ces hiérarchies est très souvent pénible pour les élèves car la sélection est objectivement construite sur l'insuffisance scolaire personnelle. Au sein d'une même classe, les enseignants, les programmes et les coefficients sont identiques pour tous. Chaque élève dispose donc, formellement, de chances égales. En conséquence, une «mauvaise * orientation est perçue par l'élève comme le fruit d'une incapacité personnelle à " avoir le niveau ". La logique de sélection est par ailleurs très largement identifiée au règne impérial de certaines matières. Le privilège des mathématiques et de la physique se révèle unanimement dénoncé, d'autant plus que ces matières prétendent évaluer objectivement les élèves.

Quant aux procédures de sélection, elles sont vécues comme brutales et autocratiques à travers un conseil de classe que la majorité des élèves perçoit comme un tribunal et une mascarade de justice : « Tout est joué d'avance et un élève n'a jamais raison $\%$. Ce discours, que l'on jugera peut-être excessif, est bien souvent partagé par les parents, les enseignants et l'administration. Personne ne semble contrôler cette sélection implacable et tout le monde la dénonce.

2. Le processus de sélection engendre une très nette séparation entre le souci d'efficacité scolaire et l'intérêt intellectuel. Les élèves, à l'exception des * héritiers \#, ne perçoivent pas le contenu culturel et éducatif de leur formation car il leur faut avant tout "assurer " pour essayer d'atteindre les filières les plus prestigieuses. Comme il importe de travailler de la manière la plus efficace et la plus productive possible, la rentabilité supplante l'intérêt comme critère scolaire. Les matières les plus sélectives, les mathématiques et la physique, sont d'ailleurs les matières les moins intéressantes et les moins prestigieuses aux yeux des élèves. C'est donc avant tout l'importance du coefficient qui commande la 
hiérarchie des efforts fournis. Les élèves se conduisent souvent comme de petits entrepreneurs, soucieux de maximiser le rapport entre le coût et les gains du travail effectué : "On laisse aller dans certaines matières secondaires pour mettre le paquet dans d'autres plus fondamentales *; on brille avant le conseil de classe pour donner une bonne image de soi. L'instrumentalisme est si fort que les élèves de seconde, quand ils sont à peu près certains de leur future orientation, jugent inutile, et parfois injuste, de devoir continuer à travailler des matières qu'ils n'auront plus au programme en première. Ce sentiment est également partagé par les élèves de la section $G$ visà-vis de la philosophie et par les élèves des lycées professionnels à l'égard des matières de l'enseignement général. Dans ce contexte où il devient primordial de réussir dans les matières qui "payent ", la fraude et la " pompe " sur la copie du voisin sont très largement considérées par les élèves comme des stratégies acceptables, à condition toutefois qu'elles ne bouleversent pas les différences de performance entre élèves au sein d'une même classe: « Frauder pour passer de 8 à 10, ça va, mais frauder pour passer de 8 à 15, ça ne se fait pas $n$. L'instrumentalisme limite tout naturellement le développement d'une intelligentsia lycéenne. Les passions et les intérêts intellectuels se jouent en effet principalement à l'extérieur de l'école et on vit d'autant mieux son expérience lycéenne qu'on s'y intéresse peu. Le rapport aux enseignants est ainsi uniquement vécu comme une série de rencontres aléatoires, comme une affaire de personnalités. C'est en quelque sorte " au hasard " des situations que l'enseignant intéresse l'élève. Les lycéens et les professeurs se reprochent d'ailleurs mutuellement cette séparation entre l'efficacité et l'intérêt: les élèves pensent que les enseignants n'ont plus " la vocation ", tandis que les professeurs estiment que les élèves ne raisonnent plus qu'en termes d'orientation et d'examen. Le cas des sections scientifiques représente en l'occurrence le meilleur exemple des effets pervers induits par ce système. Si les élèves de $\mathrm{C}$ constituent une aristocratie scolaire, ils ne forment pas une aristocratie scientifique. Ils sont en $C$ car c'est la filière la plus rentable, celle qui ouvre le plus de portes. On va en $C$ pour intégrer une prépa littéraire, HEC, un IUT ou un BTS, très rarement pour effectuer de longues études scientifiques.

3. Le trait dominant de l'expérience lycéenne est la dissociation de la personnalité et de l'école. En ce sens, l'école ne constitue plus une instance de socialisation, c'est-à-dire une institution construite sur des valeurs culturelles qui fabrique des rôles sociaux que les acteurs reproduisent ensuite dans la société. En dehors de la classe, l'école ne cherche plus en effet à contróler les conduites des élèves. Leurs vêtements, leurs coiffures, leurs comportements et leurs goûts sont considérés comme relevant d'un strict domaine privé. L'époque des
" surgés » et des "lycées casernes » appartient au passé et le lycée est devenu un espace de liberté où le contrôle social se révèle très faible. Les “couples" se tiennent par la main et s'embrassent, on peut fumer, les études ne sont plus obligatoires et on peut sortir entre les cours pour aller se détendre au * milk bar * ou au café de son choix. Cependant, le lycée ne s'est pas pour autant transformé en espace démocratique dans la mesure où cette liberté ne se traduit pas en droits : « On a des libertés mais pas de droits $\%$. disent les lycéens, et chacun sait bien qu'une liberté qui n'est pas encadrée par la loi demeure une liberté non satisfaisante. L'absence de règles interdit en effet toute négociation en cas de conflit avec l'administration ou les enseignants. Si les élèves déplorent cette situation, ils n'éprouvent néanmoins pas le désir de s'investir et de participer au système. Les militants de la FIDL éprouvent d'ailleurs beaucoup de difficultés à mobiliser les lycéens sur le thème de leurs droits. On ne veut pas s'investir car la * vraie vie » est ailleurs, dans les livres, au cinéma, dans la bande de copains ou bien au bal du samedi soir. En dehors de la classe, le lycée marque donc très faiblement les conduites des jeunes tant ses capacités d'intégration sociale sont faibles. On va au lycée mais on ne devient jamais vraiment lycéen. C'est l'extérieur qui structure les représentations et les comportements. Le lycée transmet des connaissances et sélectionne des éleves, mais il n'est plus un espace d'éducation au sens durkheimien du terme. Ce système d'organisation ne construit d'ailleurs pas un établissement scolaire, il construit plutôt des établissements duels : les cours et la sélection côté enseignants, la liberté côté cour de récréation. La faiblesse du contrőle social constitue peut-être en fait la meilleure manière d'accompagner la sélection scolaire. Si les règles de fonctionnement hors de la classe étaient identiques à celles d'il y a trente ans, il est en effet probable que le système imploserait. Mais les lycéens paient cette liberté au prix fort. Elle se traduit en effet par l'indifférence et l'anonymat. Les élèves ne se connaissent pas en dehors du petit groupe d'amis et le lycée devient un a selfservice ». Si c'est toujours à l'école que se forme la personnalité, ce n'est donc plus l'école qui forme la personnalité. L'école forme des individus plus que des acteurs sociaux, mais elle les forme * malgré elle *, en leur laissant des espaces privés et des sphères d'autonomie très larges. Trois raisons majeures semblent à l'origine de ce phénomène. En premier lieu, l'école a très probablement détruit son image d'une école égalitaire et porteuse de valeurs universelles, en devenant le principal agent de la sélection. Finalement, l'école a été une institution de socialisation tant que la sélection lui était extérieure, sociale avant d'être scolaire. En second lieu, et les enseignants le disent bien, l'école n'a plus aujourd'hui le monopole de la culture légitime. Les sources culturelles sont nombreuses et l'école est devenue un simple outil 
sur un marché de la connaissance de plus en plus concurrentiel. L'école s'adapte peut-être enfin, tout simplement, à l'évolution de la société. Comment pourrait-elle en effet s'organiser autour d'un projet culturel fort, au moment méme où la société ne propose plus cette représentation indiscutée de la culture légitime?

Cette présentation sommaire des expériences scolaires des lycéens laisse de cóté un certain nombre de thèmes. L'un d'eux est relatif à la formation, ou plus exactement a l'absence de l'action collective chez des éléves souvent mécontents en même temps que détachés. Alors que parfois la situation des élèves est difficile matériellement, que l'avenir apparaît sombre à beaucoup et que la sélection et la relation pédagogique semblent engendrer un mépris endémique, les élèves s'adaptent ot se retirent, sont peu sensibles aux tentatives de mobilisation par une élite militante et agissent surtout par explosions, par * débordements * retombant très vite, exprimant un « malaise ^ plus qu'ils ne construisent une revendication et un mouvement.

II aurait aussi fallu parler plus précisément de la relation pédagogique qui est au cour de l'expérience scolaire; notons simplement que les enseignants développent souvent des conduites très parallèles et complémentaires à celles des élèves : on y retrouve la méme sensibilité au mépris et la même distance au rôle, notamment par la très nette séparation du métier et du statut, de la définition personnelle et intime du métier et de la conception bureaucratique et «bloquée * de la place dans l'organisation. Enfin, il aurait fallu dire un mot du rôle des établissements et des stratégies propres, des a arrangements * que chacun d'eux élabore et construit pour répondre à sa situation sur le a marché » scolaire et au système de ses relations internes.

Il ressort de cette étude que le lycée ne peut plus être considéré comme une institution commandée par un modele culturel, des valeurs et une organisation centrale, comme les collèges des Jésuites ou les lycées de la République. Du point de vue des élèves, et l'on admettra qu'il peut ne pas manquer d'intérêt, l'école apparaît comme le lieu où se croisent trois logiques relativement indépendantes et dissociées : une logique d'adaptation à l'environnement répondant à une * demande scolaire *, une logique de sélection et de hiérarchisation interne, et une logique d'éducation, de culture, se jouant dans une relation pédagogique perçue comme aléatoire, et dans la vie juvénile abritée dans le lycée, permises par l'établissement, mais qui ne'commandent pas l'expérience des élèves. Aussi les élèves apparaissent-ils comme des jeunes et des individus qui vont à l'école et en usent comme ils peuvent, beaucoup plus que comme des lycéens définis par des rôles et des modes de conduites fixés. L'intégration et la socialisation laissent place à des modes d'adaptation plus ou moins heureux.

II ne faudrait certainement pas juger ces brèves remarques et observations du point de vue pessimiste d'une " crise " de l'école, comme si le déclin d'une institution de socialisation ne pouvait engendrer que la souffrance, l'anomie et le règne des intérêts égoistes. Le lycée reçoit des individus qui s'y transforment selon des processus qu'il ne contrôle guère, plus qu'il n'accueille des enfants dont il ferait des « hommes * selon un modèle culturel et un style pédagogique contrôlés et acceptés par tous. L'école gère avec plus ou moins de bonheur des exigences contradictoires et en conduisant les élèves à se vivre comme des personnes peu engagées dans ses jeux, elle s'adapte plus qu'on ne le croit souvent aux mutations sociales et culturelles de notre société.

François DUBET, Olivier COUSIN, Jean-Philippe GUILLEMET

CADIS, EHESS

Université de Bordeaux II
(1) II s'agit des travaux de Pierre Bourdieu et de son Ecole. Sur la sous-culture délinquante, cf. D. Hargreaves et al., Deviance in the claserooms, London: Routledge and Kegan Paul, 1975 ; P. Woods, 8choots and doviance, Milton Keynes : Open University Press, 1981.

(2) Cette perspective est illustré en France par les travaux de R. Boudon, L'insogallt des chances, Paris: Armand Colin, 1973.

(3) C'est par exemple l'analyse de M. Cherkaoui sur l'émergence de la sélection par les mathématiques, Les changements du gyotime colucatif on France, Paris : PUF, 1982.

(4) Cf., pour une présentation critique et complète, J.L. Forquin,
"La "nouvelle sociologie de l'éducation " en Grande-Bretagne: orientations, apports thériques, evolution (19701980) «, Rovise Francaliee de Psdagogio, 63, 1983, pp. 61-79.

(5) Sur l'hétérogénéité des publics de LP, cf. C. Baudelot et al., - Les élèves de LP, anatomie d'une population „, Revis frengaice des Affaires Soctales, déc. 1987, pp. 79-98.

(6) Le LP se distingue des CET étudiés au milieu des années soixante par. Claude Grigon, car les CET accueillaient une élite relative, beaucoup de jeunes entrant directement en apprentissage. Le LP reçoit tous les éléves qui n'ont pu se glisser dans les autres filières. Cf. L'ordre dos choses. Les fonctions eocteles de Peneolgnement technique, Paris: Ed. de Minuit, 1971. 